\title{
WEAK AND MAGNETIC INELASTIC SCATTERING OF ANTINEUTRINOS ON ATOMIC ELECTRONS
}

\author{
S.A. Fayans, L.A. Mikaelyan, V.V. Sinev \\ Russian Research Centre - "Kurchatov Institute", Kurchatov Sq. 1, \\ 123182 Moscow, Russia
}

\begin{abstract}
Neutrino scattering on electrons is considered as a tool for laboratory searches for the neutrino magnetic moment. We study inelastic $\bar{\nu}_{e} e^{-}$-scattering on electrons bound in the germanium $(Z=32)$ and iodine $(Z=53)$ atoms for antineutrinos generated in a nuclear reactor core and also in the ${ }^{90} \mathrm{Sr}-{ }^{90} \mathrm{Y}$ and ${ }^{147} \mathrm{Pm}$ artificial sources. Using the relativistic Hartree-Fock-Dirac model, we calculate both the magnetic and weak scattering cross sections for the recoil electron energy range of 1 to $100 \mathrm{keV}$ where a higher sensitivity to the neutrino magnetic moment could be achieved. Particular attention is paid to the approxi-mate procedure which allows us to take into account the effects of atomic binding on the inelastic scattering spectra in a simple way.
\end{abstract}

\section{INTRODUCTION}

In the present paper some issues of the low-energy neutrino physics are considered that could be essential for the current and future experiments aimed to search for a "large" neutrino magnetic moment. In the previous publication [1] we have studied the inelastic weak and magnetic scattering of reactor 
antineutrinos on the $\mathrm{K}$ - and L-shell electrons in a iodine atom. Here we extend our calculations to the iodine M-shell electrons, and present the results for the K- and L-shell electrons bound in a germanium atom. Besides the reactor $\bar{\nu}_{e}$ 's, we also consider electron antineutrinos emitted by the ${ }^{90} \mathrm{Sr}-{ }^{90} \mathrm{Y}$ and ${ }^{147} \mathrm{Pm}$ artificial sources. The use of artificial $\bar{\nu}_{e}$ sources and semiconductor germanium detectors in experiments on the neutrino magnetic moment has been considered in a number of recent publications [2, 3] (and references therein).

For the reactor $\bar{\nu}_{e}$ 's, as was shown in ref. [1], the free scattering differential cross sections can be approximately converted to the inelastic ones by means of a simple step-function transformation. Here we investigate the accuracy of such a recipe in more detail, with different $\bar{\nu}_{e}$ energy spectra.

As an input, we use the standard power reactor $\bar{\nu}_{e}$ spectrum corrected for antineutrinos from the beta-emitters born in a core through the $(n, \gamma)$ reactions [4]; for the ${ }^{90} \mathrm{Sr}-{ }^{90} \mathrm{Y}$ and ${ }^{147} \mathrm{Pm}$ sources we use the spectra tabulated in ref. [5]. The chosen input spectra are plotted in Fig. 1. The calculated recoil electron energy spectra with these three sources for the case of a free $\bar{\nu}_{e} e^{-}$-scattering are presented in Fig. 2. In these calculations, eqs. (2)-(4) of ref. [1] have been used. The free magnetic- and weak-scattering recoil spectra are denoted as $S_{\text {free }}^{M}$ and $S_{\text {free }}^{W}$, respectively. All calculations in the present paper are done for the neutrino magnetic moment $\mu=2 \cdot 10^{-11} \mu_{\mathrm{B}}$ $\left(\mu_{\mathrm{B}}\right.$ is the Bohr magneton), the constants of the electroweak interaction are the same as in ref. [1].

\section{INELASTIC SCATTERING ON ATOMIC ELECTRONS}

In this section we consider the $\bar{\nu}_{e} e^{-}$scattering on electrons bound in the iodine and germanium atoms. The energies and wave functions of the discrete single-electron states are calculated within the relativistic self-consistent Hart-ree-Fock-Dirac (HF-D) approach, with a local exchange-correlation potential. The wave functions of outgoing electrons in the continuum were obtained by a numerical integration of the Dirac equation in the HF-D mean field. The details of this approach can be found in refs. [6]. The calculated energies of some electronic subshells are listed in Table 1, they agree with 
Table 1. Calculated HF-D binding energies (in keV) of some electronic shells in iodine and germanium atoms

\begin{tabular}{c|c|ccc|cccccc}
\hline & \multicolumn{10}{|c}{ Shell } \\
\multirow{2}{*}{ Atom } & $\mathrm{K}$ & $\mathrm{L}_{\mathrm{I}}$ & $\mathrm{L}_{\mathrm{II}}$ & $\mathrm{L}_{\mathrm{III}}$ & $\mathrm{M}_{\mathrm{I}}$ & $\mathrm{M}_{\mathrm{II}}$ & $\mathrm{M}_{\mathrm{III}}$ & $\mathrm{M}_{\mathrm{IV}}$ & $\mathrm{M}_{\mathrm{V}}$ \\
\cline { 2 - 10 } & & $1 \mathrm{~s}_{1 / 2}$ & $2 \mathrm{~s}_{1 / 2}$ & $2 \mathrm{p}_{1 / 2}$ & $2 \mathrm{p}_{3 / 2}$ & $3 \mathrm{~s}_{1 / 2}$ & $3 \mathrm{p}_{1 / 2}$ & $3 \mathrm{p}_{3 / 2}$ & $3 \mathrm{~d}_{3 / 2}$ & $3 \mathrm{~d}_{5 / 2}$ \\
\cline { 2 - 10 } $\mathrm{I}$ & 32.9 & 5.09 & 4.78 & 4.48 & 1.03 & 0.90 & 0.84 & 0.61 & 0.60 \\
$\mathrm{Ge}$ & 10.9 & 1.35 & 1.22 & 1.18 & 0.18 & & & & \\
\hline
\end{tabular}

spectroscopic data within a few percent.

For an iodine atom, the weak and magnetic inelastic $\bar{\nu}_{e} e^{-}$-scattering cross sections have been calculated for K-, L- and M-shells which contain in total 28 electrons, the remaining 25 electrons with binding energies less than 200 $\mathrm{eV}$ were considered as free. For germanium, atomic binding of K- and L-shell electrons has been taken into account, all the other electrons were treated as free. We shall denote the inelastic recoil energy spectra for electrons knocked out from an atom due to the magnetic and weak interactions by $S_{\mathrm{in}}^{M}$ and $S_{\mathrm{in}}^{W}$, respectively.

Shown in the top panels of Figs. 3 and 4 are the results of calculations of the recoil spectra $S_{\mathrm{in}}^{M}$ and $S_{\mathrm{in}}^{W}$, respectively, for electrons knocked out from different electronic subshells of an iodine atom by $\bar{\nu}_{e}$ 's from the ${ }^{90} \mathrm{Sr}-{ }^{90} \mathrm{Y}$ source. In the bottom panels in these figures, the ratios to the corresponding free recoil spectra are plotted. It can be seen that the magnetic scattering is strongly suppressed by the atomic binding effect for the K- and L-shell electrons, and even for the M-shell with lower binding energy, the effect of suppression is quite noticeable. Atomic electron binding also influences the 
weak scattering cross sections but the effect is much weaker. This unfavours the detectability of the neutrino magnetic moment.

As follows from our calculations, the ratios $S_{\text {in }}^{M, W}(T) / S_{\text {free }}^{M, W}(T)$ for the other sources and target atoms are typically of the same character as those presented in Figs. 3 and 4.

Let us introduce now, instead of the electron kinetic energy $T$, the energy transfer $q$ which is defined by

$$
q=\Delta E=\epsilon_{i}+T
$$

where $\Delta E$ is the neutrino energy loss in the inelastic scattering process, $\epsilon_{i}$ is the electron binding energy on the considered atomic shell (the nuclear recoil is neglected). For the scattering on free electrons one has $\epsilon=0$ and $q=T$. In practice, for majority of detectors, $q$ is just the total energy that could be recorded as a visible energy of the event since the soft X-rays and Auger electrons, ejected to fill the vacancy in the shell, are absorbed in the detector sensitive volume and their summed energy, i.e. $\epsilon_{i}$, is added to the kinetic energy $T$ of the recoil electron.

It has been demonstrated in ref. [1] that, for reactor antineutrinos, the inelastic scattering spectrum $S_{\text {in }}^{i}(q)$ for electrons from the subshell $i$ of the iodine atom can be obtained from the free scattering spectrum $S_{\text {free }}(q)$, taken at the same visible energy $q$, if one introduces the response function $R^{i}\left(q, \epsilon_{i}\right)$ defined by the relation

$$
S_{\text {in }}^{i}(q)=R^{i}\left(q, \epsilon_{i}\right) \times S_{\text {free }}(q)
$$

in which $R^{i}\left(q, \epsilon_{i}\right)$ is approximated by the Heaviside step-function $\theta\left(q-\epsilon_{i}\right)$ :

$$
R^{i}\left(q, \epsilon_{i}\right) \approx \theta\left(q-\epsilon_{i}\right)= \begin{cases}1, & q>\epsilon_{i} \\ 0, & q<\epsilon_{i} .\end{cases}
$$

In the following, this will be referred to as the zero (or step-function) approximation.

Now, for each antineutrino source under consideration, we calculate the "exact" spectra $S_{\text {in }}^{i}(q)$ of electrons knocked out from the iodine and germanium atomic subshells in the magnetic and weak $\bar{\nu}_{e} e^{-}$-scattering processes, evaluate the "exact" partial response functions $R^{i}\left(q, \epsilon_{i}\right) \equiv S_{\text {in }}^{i}(q) / S_{\text {free }}^{i}(q)$ 
and analyze their deviations from the zero approximation of eq. (3). "Exact" means that the calculations are performed with the HF-D model. Some of the obtained results are discussed below.

For the L- and M-shell electrons, as can be seen in Figs. 5 and 6, the "exact" response functions both for magnetic and weak inelastic scattering processes differ only slightly one from the other. They, however, are close to unity everywhere except the rather narrow regions near the corresponding threshold energies $q=\epsilon_{i}$. The same is true, in case of magnetic scattering, for the K-shell electrons; on the other hand, the weak scattering K-shell response function exceeds unity at sufficiently large energies. This latter effect reflects the influence of the atomic binding at relativistic energies [7] due to which the weak inelastic cross section could be enhanced by a factor of the order of $1+O\left(\alpha^{2} Z^{2}\right) \sim 1+O\left(\epsilon_{K} / m c^{2}\right)$ ( $\alpha$ is the fine structure constant, $m$ the electron mass, $\epsilon_{K}$ the K-shell binding energy); such an effect might be of relative importance only for the tightly bound K-shell electrons.

For the soft ${ }^{147} \mathrm{Pm}$ antineutrinos, as seen in Figs. 7 and 8, the zero approximation does not provide a good fit for the weak scattering on the K-shell electrons either in iodine or germanium atom. We also note that the stepfunction approximation is strongly violated at the energy transfers around the free scattering upper limit $q^{\max }=2 E^{2} /\left(2 E+m c^{2}\right)(E$ is the incoming

$\bar{\nu}_{e}$ energy). For $q>q^{\max }$, the free scattering cross sections are identically zero while the inelastic ones are small but still finite in the "kinema-tically forbidden" region beyond $q^{\max }[6,7]$. This tendency can be clearly seen for all iodine and germanium subshells: for the energy transfer $q>80 \mathrm{keV}$, as demonstrated in Figs. 7-9, the ratios $S_{\text {in }}^{i}(q) / S_{\text {free }}^{i}(q)$ rapidly increase with $q$.

\section{HOW GOOD IS THE STEP-FUNCTION APPROXIMATION?}

To obtain an "exact" visible-energy-transfer electron spectrum $S_{\text {in }}^{Z}(q)$ for a given atom, we have to sum all the partial contributions $S_{\text {in }}^{i}(q)$ from different subshells:

$$
S_{\text {in }}^{Z}(q)=\sum\left(n_{i} / Z\right) S_{\text {in }}^{i}(q)=S_{\text {free }}(q) \cdot \sum\left(n_{i} / Z\right) R^{i}\left(q, \epsilon_{i}\right)
$$

Here $n_{i}$ is the number of electrons in the $i$-th subshell; the spectrum is normalized to one electron. According to Eq. (4), the "exact" response function 
for the whole atom has the form

$$
R^{Z}(q) \equiv S_{\text {in }}^{Z}(q) / S_{\text {free }}(q)=\sum\left(n_{i} / Z\right) R^{i}\left(q, \epsilon_{i}\right)
$$

and, correspondingly, the atomic response function in the zero approximation reads

$$
R_{0}^{Z}(q)=\sum\left(n_{i} / Z\right) \theta\left(q-\epsilon_{i}\right) .
$$

One sees that $R_{0}^{Z}$ is defined only by a set of the shell energies $\epsilon_{i}$ and shell occupancies $n_{i}$ of the particular atom. It is the same for magnetic and weak scattering and does not depend on the $\bar{\nu}_{e}$ source. For the iodine and germanium atoms, the functions $R_{0}^{Z}$ are shown in Fig. 10.

To check the validity of the step-function approximation for the three $\bar{\nu}_{e}$ sources considered in the present paper, we have calculated the "exact" electron energy spectra $S_{\text {in }}^{Z}(q)$ for the weak and magnetic $\bar{\nu}_{e}$ scattering on these two atoms. We have got in total 12 different spectra. Then 12 corresponding "exact" response functions (5) have been evaluated and com-pared with those obtained in the zero approximation of Eq. (6). The main output of this comparison can be summarized as follows:

In the $q=(1.5-100) \mathrm{keV}$ energy range for the reactor and ${ }^{90} \mathrm{Sr}^{90}{ }^{90} \mathrm{Y} \bar{\nu}_{e}$ sources, and in the $q=(1.5-80) \mathrm{keV}$ range for the ${ }^{147} \mathrm{Pm} \bar{\nu}_{e}$ source, the relative deviation $\left|R_{0}^{Z}-R^{Z}\right| / R_{0}^{Z}$ does not exceed (1.5-2)\% except the case of weak scattering of ${ }^{147} \mathrm{Pm} \bar{\nu}_{e}$ 's on an iodine atom where the deviation can reach $(3-4) \%$.

\section{CONCLUSIONS}

A simple prescription is formulated on how to take into account the effects of atomic electron binding: To a good approximation, to obtain a visibleenergy single-electron spectrum for the atomic inelastic $\bar{\nu}_{e} e^{-}$-scattering, it is sufficient to multiply the free-electron spectrum by the "zero response function" $R_{0}^{Z}=\sum\left(n_{i} / Z\right) \theta\left(q-\epsilon_{i}\right)$. Such a step-function approximation can be safely used in the majority of current and future experiments aimed at searches for the neutrino magnetic moment.

We note, however, that this prescription is not universal. It can be used for some specific though practically important cases like those considered in the present paper. For much softer antineutrinos (e.g., from tritium source), 
the direct accurate calculations of the cross sections for $\bar{\nu}_{e} e^{-}$-scattering on the atomic electrons are needed.

\section{ACKNOWLEDGMENTS}

This work is supported in part by the Russian Foundation for Basic Research (RFBR), project 00-15-96708.

\section{References}

[1] Kopeikin V.I., Mikaelyan L.A., Sinev V.V., Fayans S.A. // Yad. Fiz. 1997, T. 60, P. 2032 [Phys. At. Nucl. 1997, V. 60, P. 1859].

[2] Kornoukhov V.N. // Yad. Fiz. 1998, V. 61, P. 1352 [Phys. At. Nucl. 1998, V. 61, P. 1250]; Bergelson B.R. et al. // Yad. Fiz. 1998, V. 61, P. 1347 [Phys. At. Nucl. 1998, V. 61, P. 1245]; Mikaelyan L.A., Sinev V.V.,

Fayans S.A. // Pis'ma v ZhETF 1998, V. 67, P. 435 [JETP Lett. 1998, V. 67, P. 453.]

[3] Beda A.G., Demidova E.V., Starostin A.S., Voloshin M.B. // Yad. Fiz. 1998, V. 61, P. 72; [Phys. At. Nucl. 1998, V. 61, P. 66.]

[4] Bakalyarov A., Kopeikin V.V., Mikaelyan L.A. // Yad. Fiz. 1996, V. 59, P. 1225; [Sov. J. Nucl. Phys. 1996, V. 59, P. 1171]; Kopeikin V.V., Mikaelyan L.A., Sinev V.V. // Yad. Fiz. 1997, V. 60, P. 230; [Phys. At. Nucl. 1997, V. 60, P. 172.]

[5] Reference book (in Russian) (Beta i antineitrinnoie izluchenie, Spravochnik), ed. Rubtsov P.M., Atomizdat, Moscow,(1989).

[6] Fayans S.A., Dobretsov V.Yu., Dobrotsvetov A.V. // Phys. Lett. B 1992, V. 291, P. 1. Dobretsov V.Yu., Dobrotsvetov A.B., Fayans S.A. // Yad. Fiz. 1992, V. 55, P. 2126; [Sov. J. Nucl. Phys. 1992, V. 55, P. 1180].

[7] Fayans S.A, Dobretsov V.Yu., Dobrotsvetov A.B..// in Proc. III Int. Symp. on Weak and Electromagnetic Interactions in Nuclei (WEIN-92), Dubna, 1992, ed. Vylov Ts.D. (Word Scientific), P. 773. 

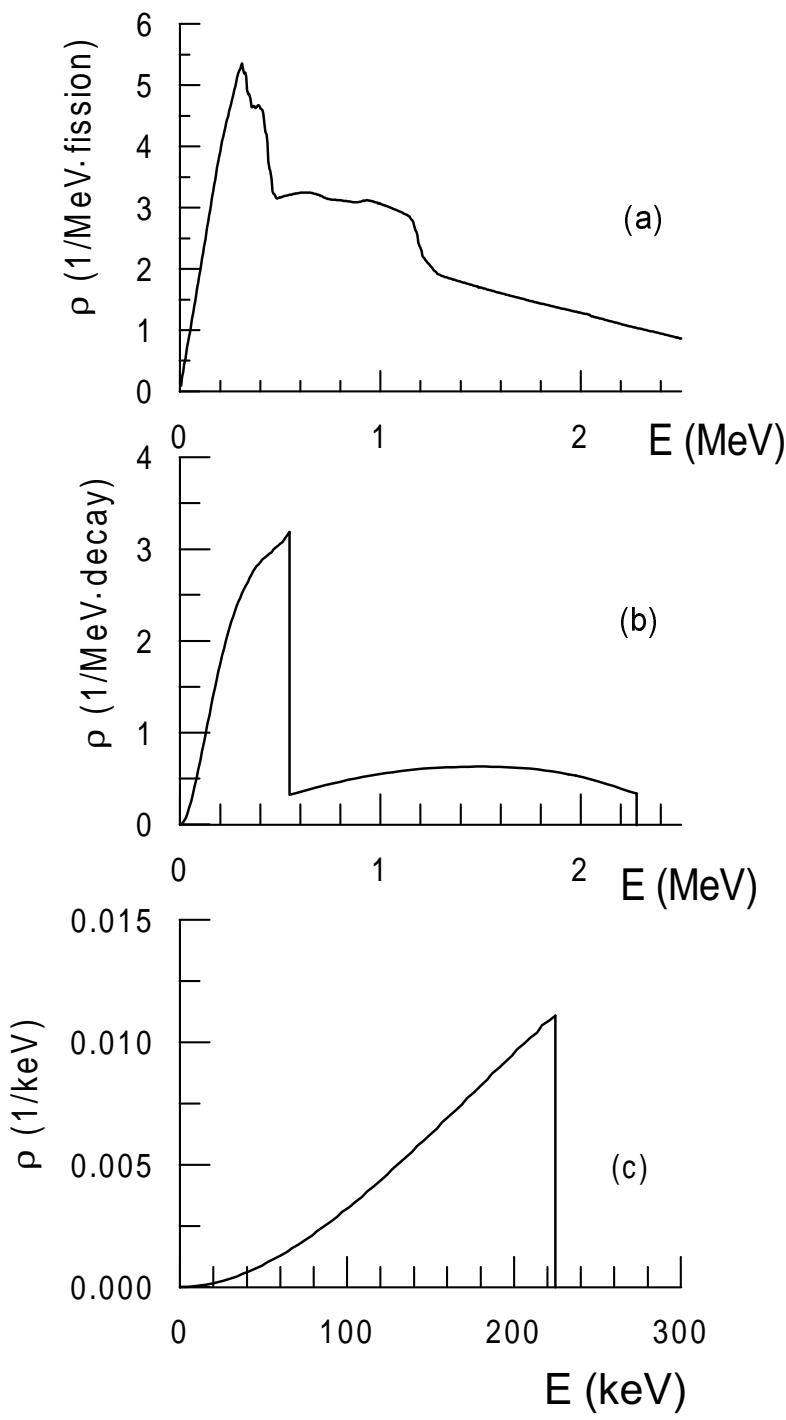

Fig. 1. Antineutrino energy spectra from three different sources:

(a) reactor $\bar{V}_{e}$ (only the low energy part is shown), (b) $\bar{\nabla}_{e}$ 's from the ${ }^{90} \mathrm{Sr}^{-90} \mathrm{Y}$ source ( two $\overline{\mathrm{V}}_{e}$ 's per sequentional ${ }^{90} \mathrm{Sr}^{90}{ }^{90} \mathrm{Y}$ decay), (c) $\bar{v}_{e}^{\prime}$ s from the ${ }^{147} \mathrm{Pm}$ source. 


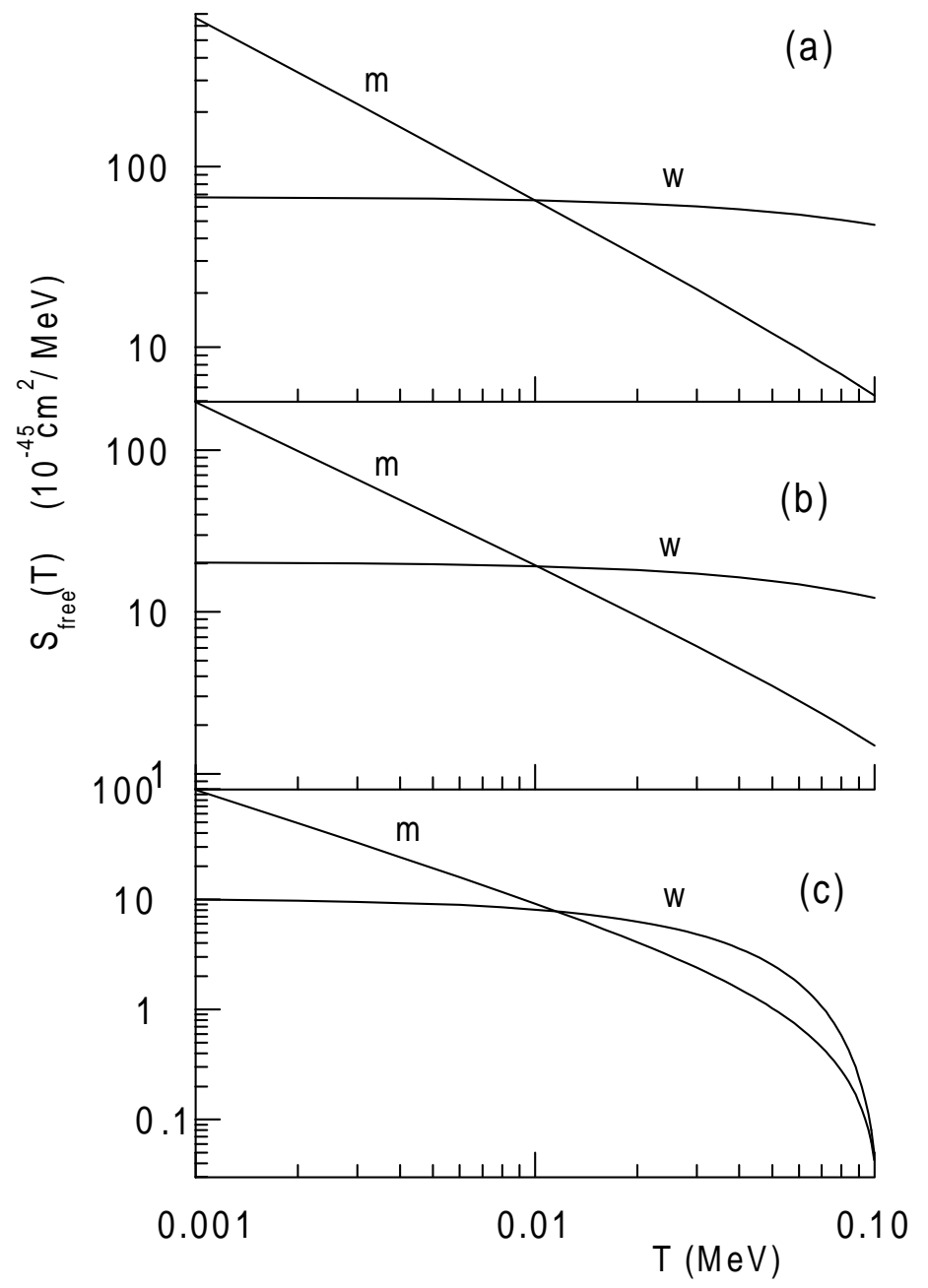

Fig. 2. Kinetic energy spectra of recoil electrons for magnetic $(\mathrm{m})$ $\left(\mu=2 \cdot 10^{-11} \mu_{\mathrm{B}}\right)$ and weak $(\mathrm{w}) \overline{\mathrm{v}}_{e} e$ - scattering on free electrons for the reactor $(\mathrm{a}), \operatorname{Sr}-\mathrm{Y}(\mathrm{b})$ and $\mathrm{Pm}(\mathrm{c})$ antineutrinos. 

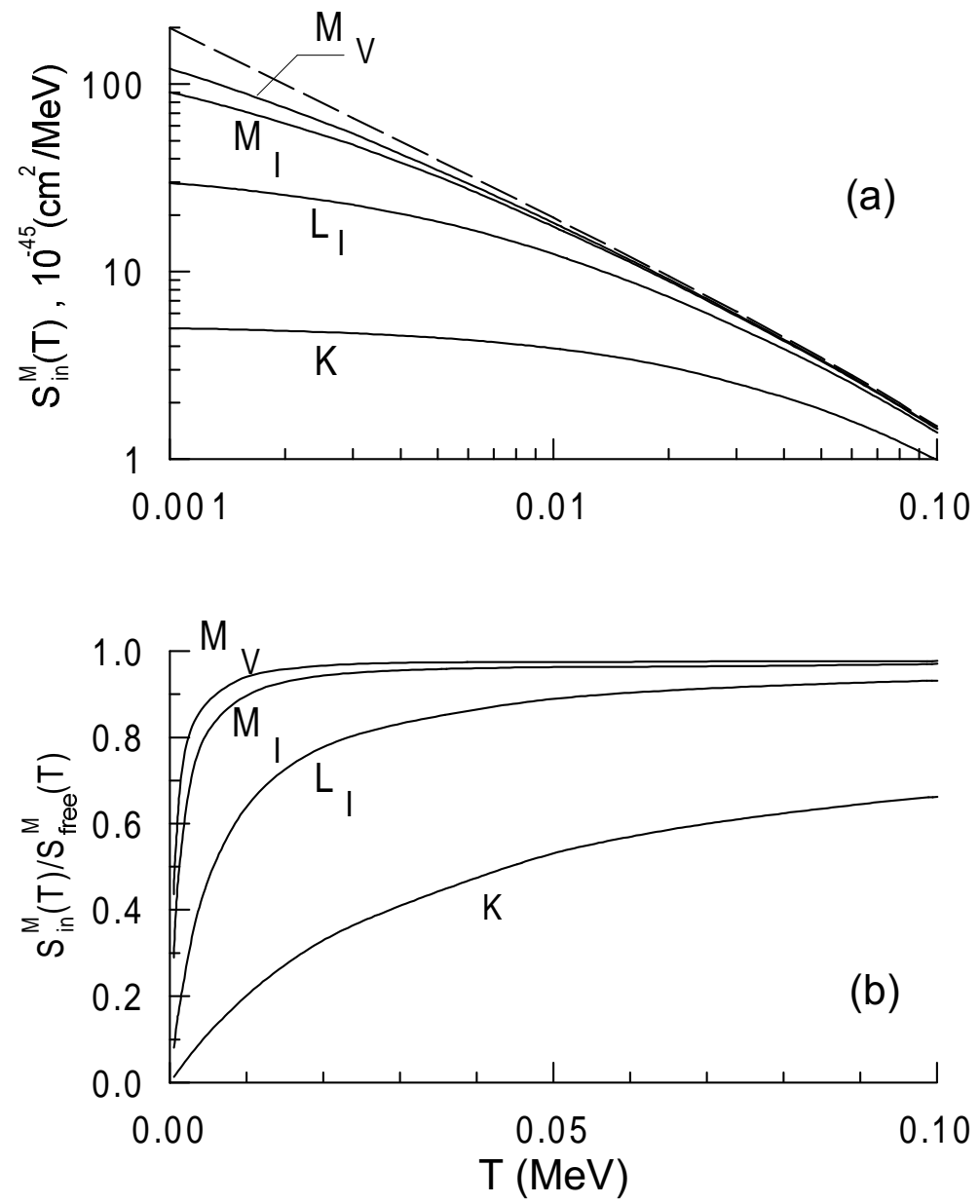

Fig. 3. Inelastic magnetic scattering of the ${ }^{90} \mathrm{Sr}^{90} \mathrm{Y}$ antineutrinos on the iodine atomic electrons vs electron kinetic energy. The curves correspond to the different subshells and are marked in the same way as in Table 1. (a) - electron kinetic energy spectra; dashed curve is for the scattering on free electrons $\left(\mu=2 \cdot 10^{-11} \mu_{B}\right)$. (b) - ratios of the inelastic spectra to the free spectrum $S_{i n}^{M}(T) / S_{\text {free }}^{M}(T)$. 

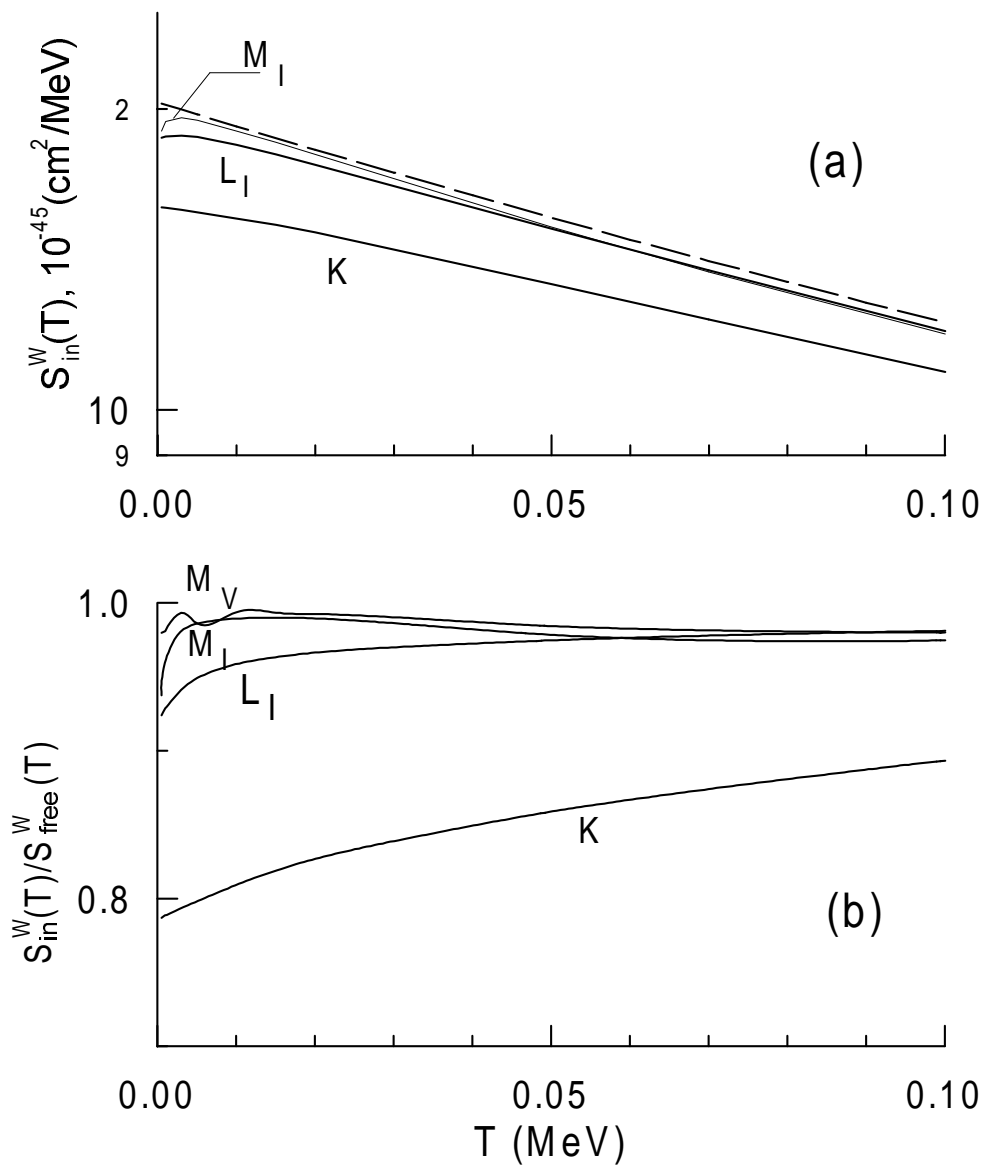

Fig. 4. The same as in Fig. 3. but for weak scattering. 


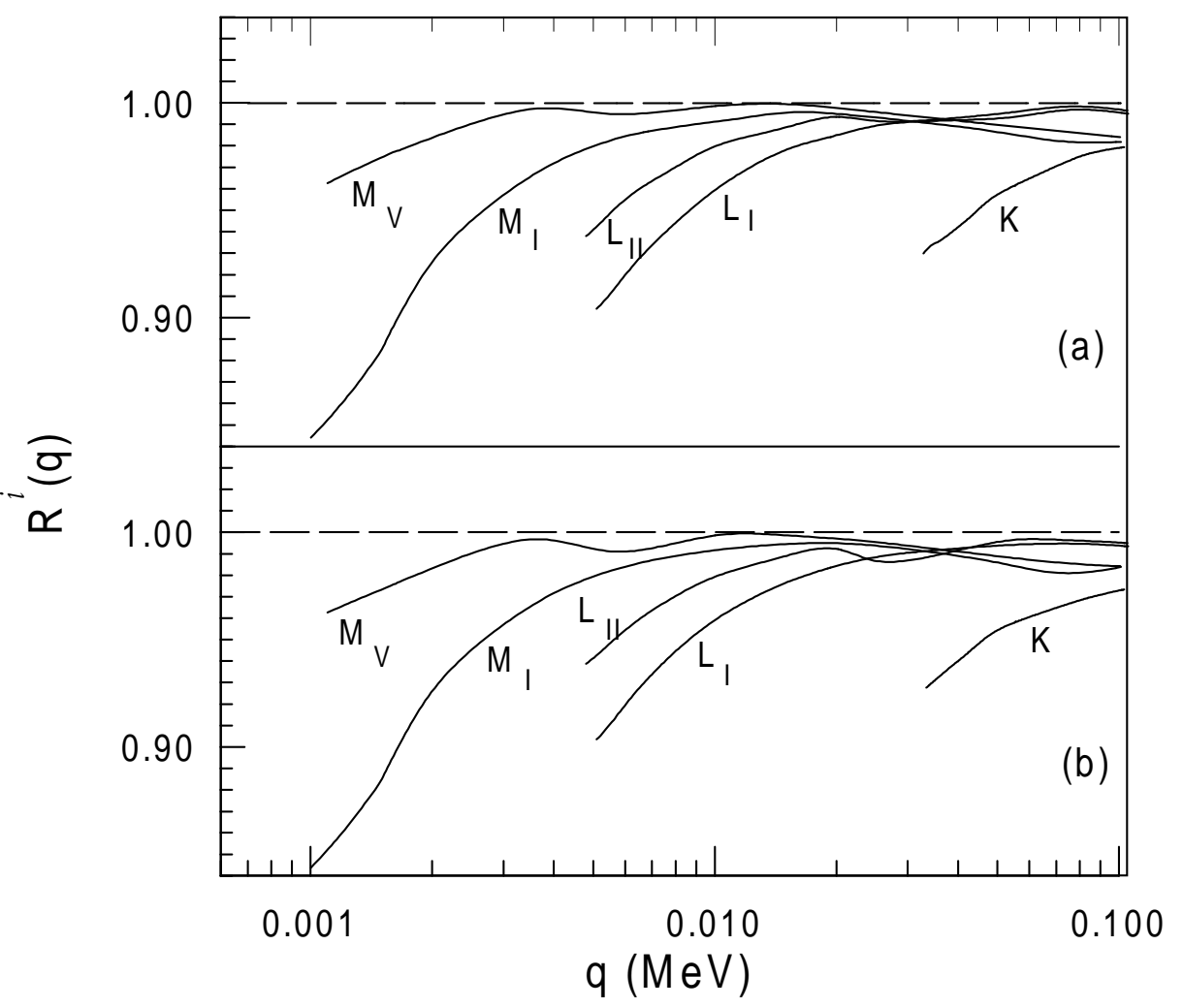

Fig. 5. "Exact" response functions $R^{i}(q)=S_{\text {in }}^{i}(q) / S_{\text {free }}(q)$ for inelastic magnetic $\bar{v}_{e} e^{-}$-scattering on the $\mathrm{K}-, \mathrm{L}$ - and $\mathrm{M}$-shell electrons of an iodine atom. The curves are marked with the corresponding subshells as in Table 1. (a) for reactor antineutrinos, (b) for $\overline{\mathrm{v}}_{e}$ 's from the ${ }^{90} \mathrm{Sr}-{ }^{9} \mathrm{Y}$ source. 


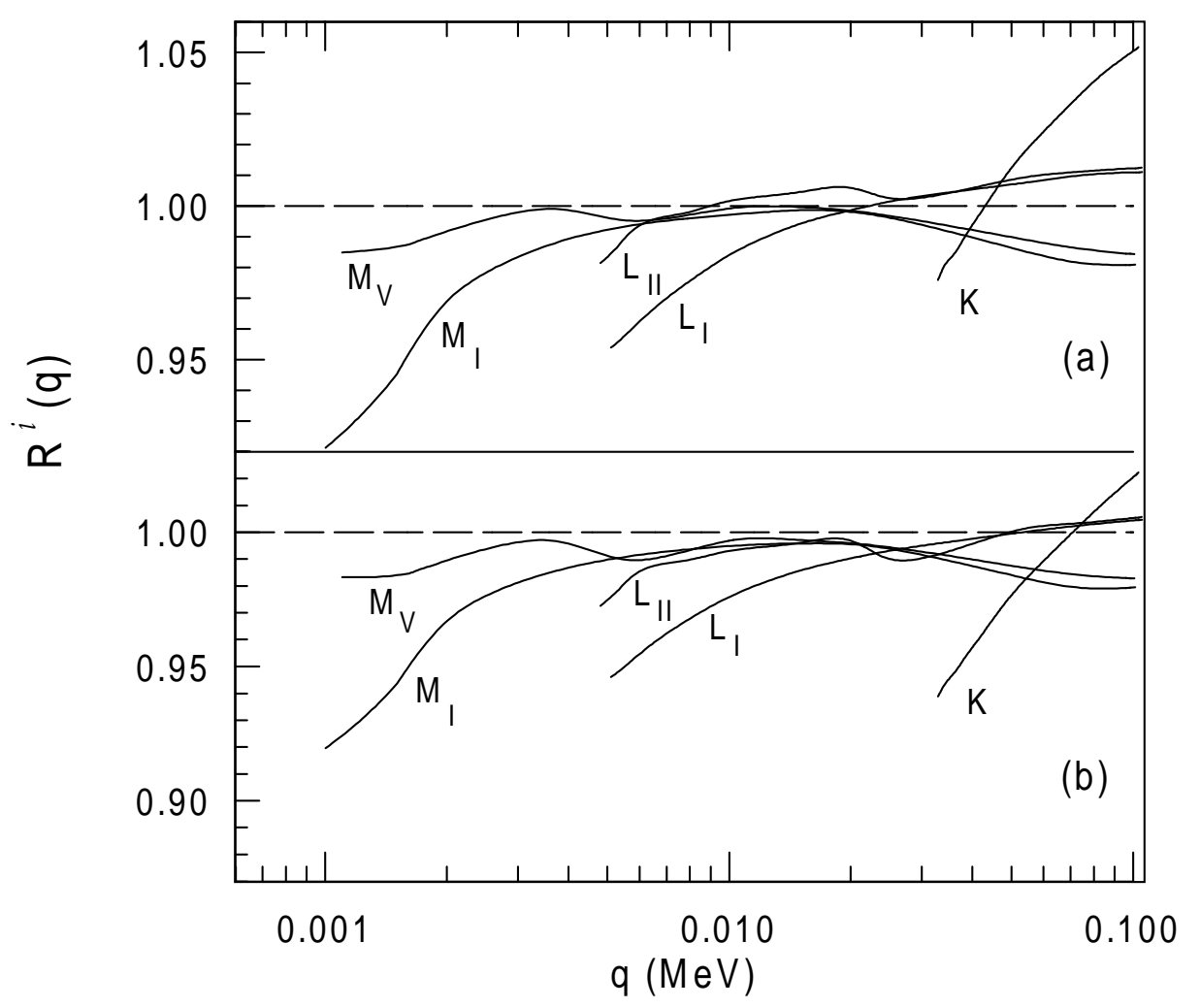

Fig. 6. The same as in Fig.5. but for weak scatterring. 


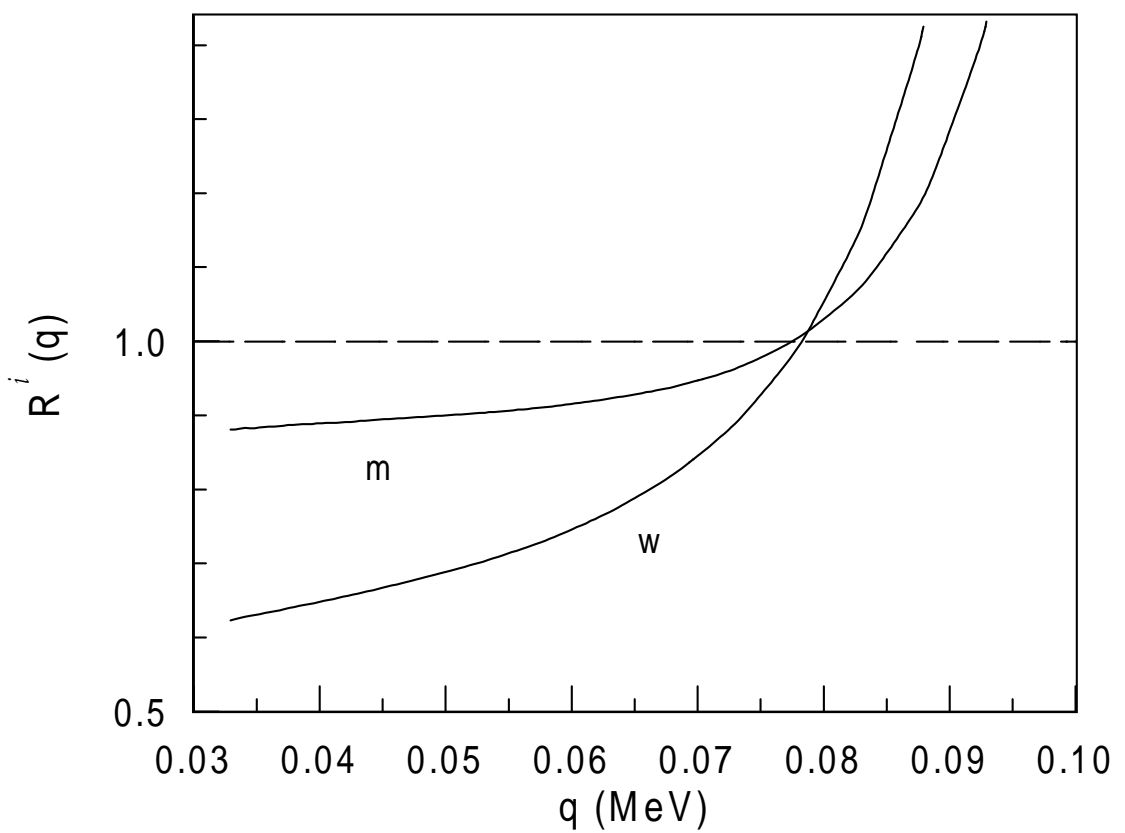

Fig. 7. "Exact" response functions for magnetic (m) and weak (w) $\bar{v}_{e} e^{-}$ scattering on the iodine $\mathrm{K}$-shell electrons for the ${ }^{147} \mathrm{Pm}$ source. 


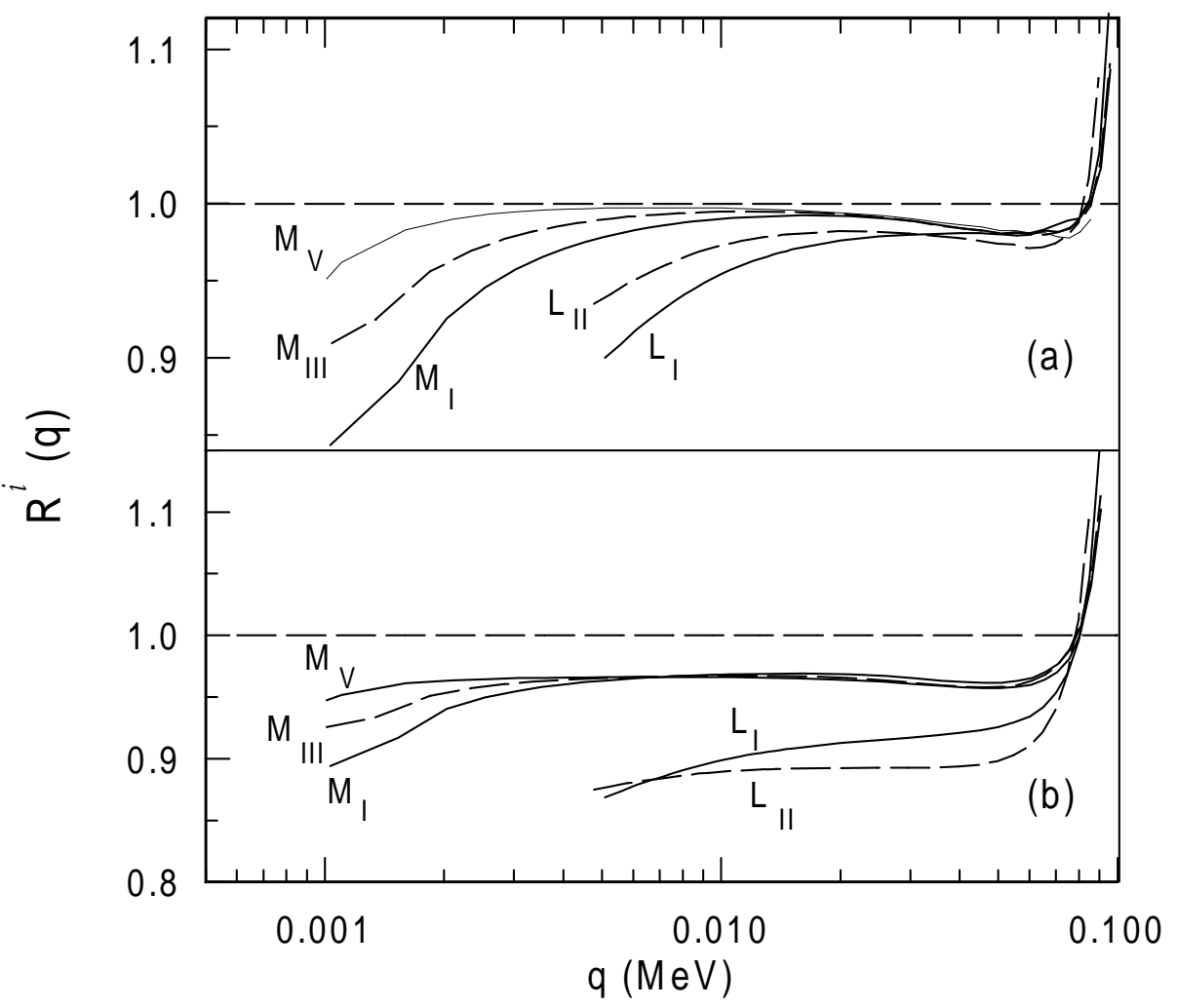

Fig. 8. "Exact" responce functions for magnetic (a) and weak (b) $\overline{\mathbf{v}}_{e} e^{-}$scattering on the iodine $\mathrm{L}$ - and $\mathrm{M}$-shell electrons for the ${ }^{147} \mathrm{Pm}$ source. The curves are marked with the corresponding subshells as in Table 1. 


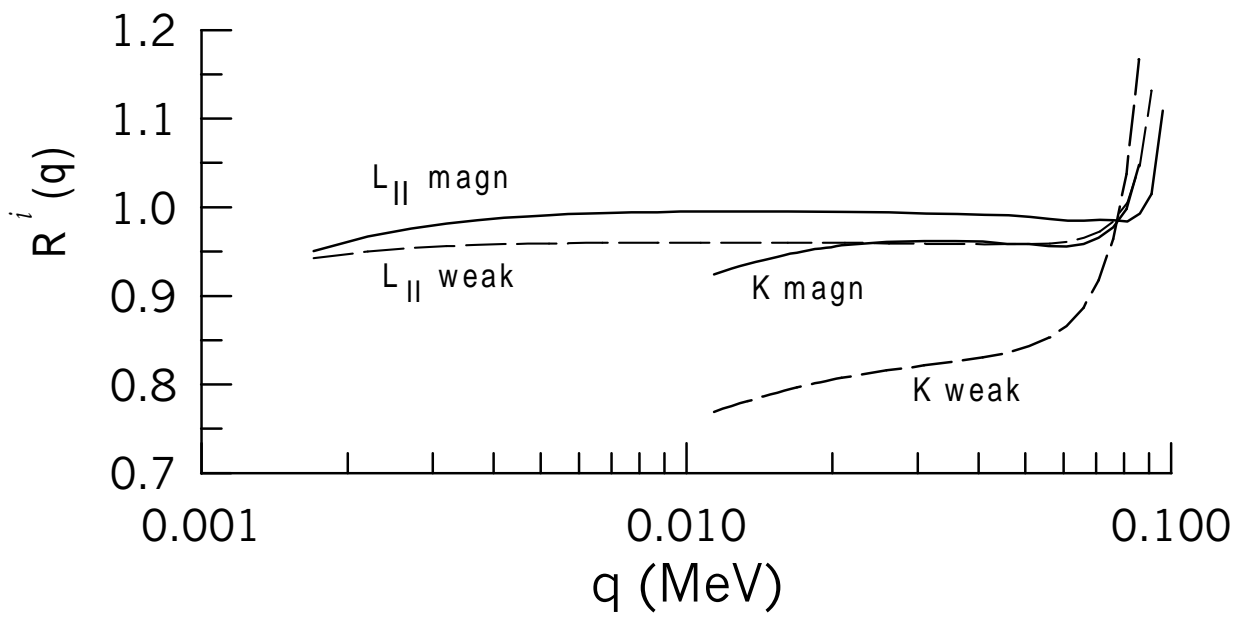

Fig. 9. "Exact" response functions for $\bar{v}_{e} e$-scattering on the germanium $\mathrm{K}$ - and L-shell electrons for the ${ }^{147} \mathrm{Pm}$ source. The curves are marked with the corresponding subshells as in Table 1. 


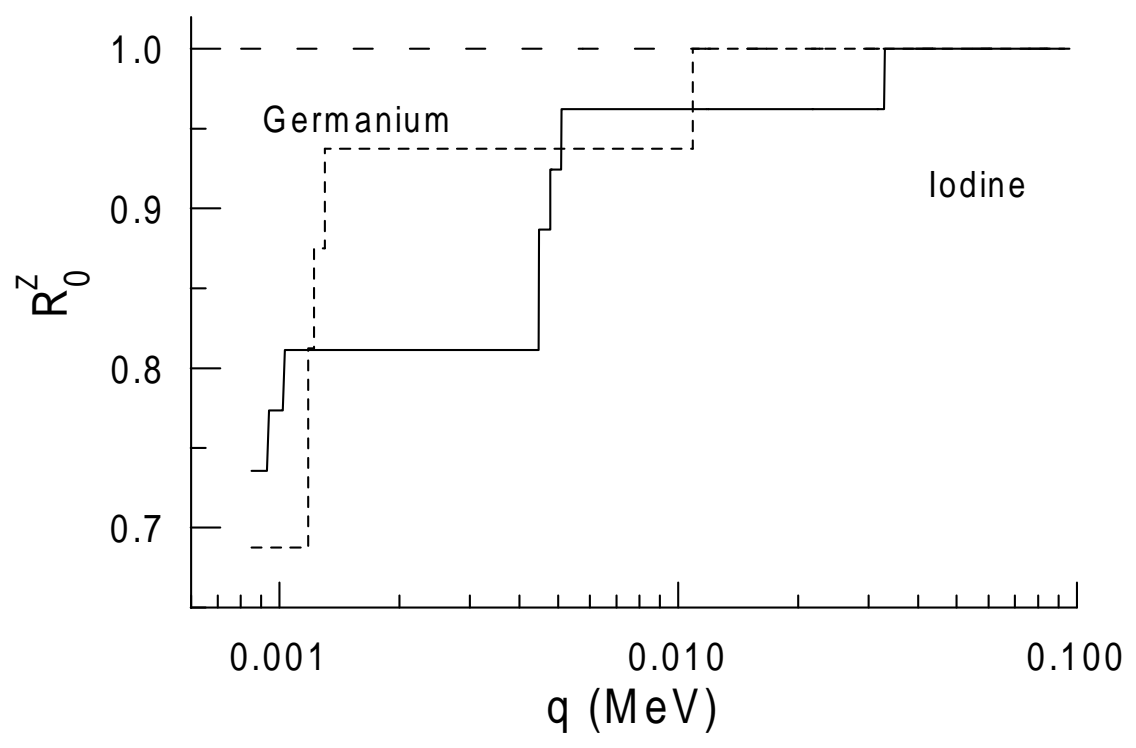

Fig. 10. Zero approximation responce functions calculated according to eq. (6) for iodine and germanium atoms. 